\title{
Advanced Architecture Algorithm of Sensor based Robotics Security System framework for e-Governance Technology
}

\author{
Rajeev Kumar \\ Department of Computer Application, \\ College of Management \& Computer Application, \\ Teerthankar Mahaveer University, Moradabad,
}

\author{
M.K. Sharma \\ Associate Professor \& Head MCA Program \\ Department of Computer Science, Amrapali \\ Institute -Haldwani (Uttarakhand)
}

\begin{abstract}
In this paper, we discuss the advanced sensor based system framework for security application is proposed in eGovernance security areas. Like online voting, online transaction, online payment and automatic transportation management system. Fidelity and stability are contradicting factors in security system. Most of security manipulations nowadays are performed manually by the human operators. In automated security system used future and high level technology which is very secure and protected and new technologies security system. These highly precise operations require high-skilled professional operators. In this we are using the artificial intelligence system and information and communication technology (ICT) that has able to develop a high protected sensor based security system framework. However, the success and survival of the networks security is very low due to the great sensitivity of security system in egovernance. In this technology ICT useful for sharing the information and communicate the human operator and operate the manually \& automatically. In added of high security we used this technology, during every security operation the human's has a certain role but sensor based system is more powerfully and reliable that can affect the quality of different security areas. All of the reasons listed above show that the system manipulation is an exclusively high security level task for a human operator to perform. So Therefore, if we applying the sensor based security system framework for the egovernance security than manipulation may provide us with many advantages.
\end{abstract}

\section{Keywords}

Advanced architecture, cloud Computing, Algorithm, Security areas, Security Issues, e-Governance Application.

\section{INTRODUCTION}

The main objective of this paper is to present a new SensorBased security system Planning framework for e-governance system robot navigation in unknown environments. The key idea of the sensor based robotics security system technique is to exploit the information obtained about the environment topology through the sensors and information and Communication technology(ICT) functions to bias the distribution of random nodes in e-governance technology -like approach towards critical regions, i.e. narrow passages and hard-to-navigate regions.

This results in a better coverage of the free space especially in environments where narrow passages exist. Inspired by the promising results obtained using this technique for modelbased cases we propose a sensor-based robotics security planning framework. [1] In our proposed sensor-based technique information obtained about the C-space topology through the FD paradigm is utilized to find the next best view configuration where each scan should be carried out. They are actually measuring the force which produces the acceleration of a known mass. Different types of acceleration transducers are known: stress-strain gage, piezoelectric, capacitive, and inductive. Micromechanical accelerometers have been developed. In this case the force is measured by measuring the strain in elastic cantilever beams formed from silicon dioxide by an integrated circuit fabrication technology. Sensors are used to detect the positive contact between two mating parts and/or to measure the interaction forces and torques which appear while the robot manipulator conducts part mating operations. [2] Another type of contact sensors is the tactile sensors which measure a multitude of parameters of the touched object surface.

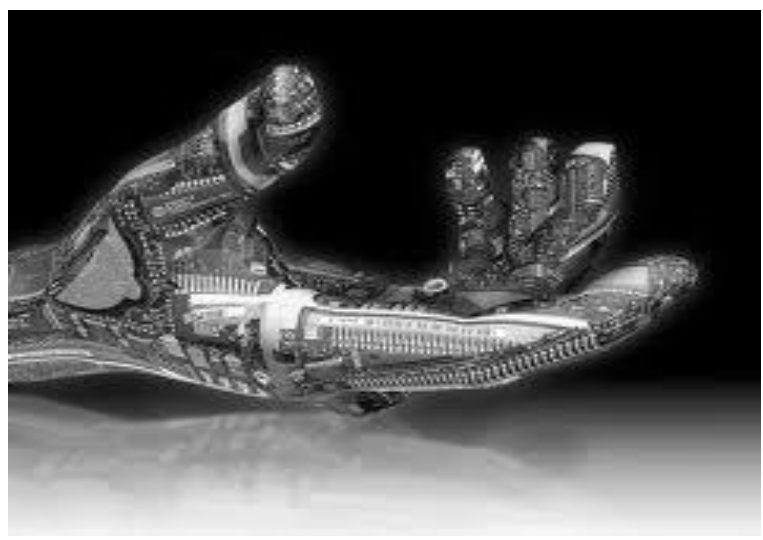

Figure 1: Robots Hands with Control

\section{RANGE OF SENSORS}

Range sensors measure the distance of two areas and objects in their operation area are used. They are used for robot navigation one contact to other contacts, in robotics system are used the sensors and sensors are specified the particular range. If robots sensors are very fares then these are not working properly and obstacle avoidance or to recover the third dimension for monocular vision. Range sensors are based on one of the two principles: time-of-flight and triangulation. [3]

Time-of-flight sensors estimate the range by measuring the time elapsed between the transmission and return of a pulse. Laser range finders and sonar are the best known sensors of this type. 
Triangulation sensors measure range by detecting a given point on the object surface from two different points of view at a known distance from each other. Knowing this distance and the two view angles from the respective points to the aimed surface point, a simple geometrical operation yields the rance [3]

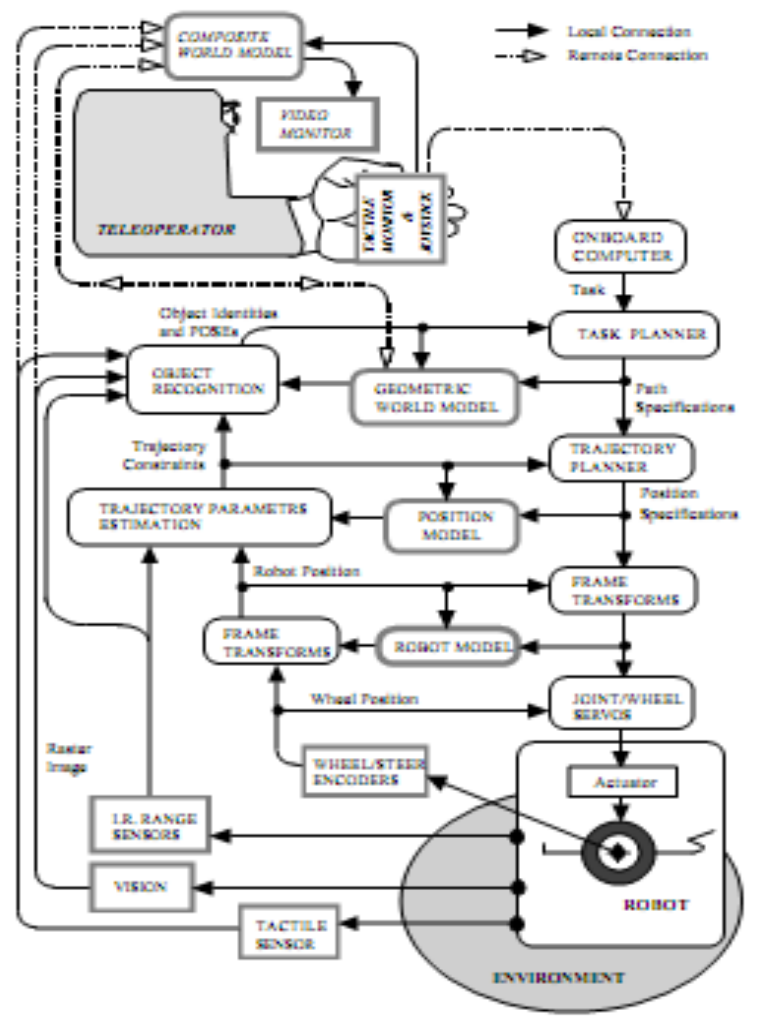

Figure 2: Control System for e-Governance

\subsection{Robot controller can have a multi-level}

hierarchical architecture: these are the level of

Robot controller as follows:

\subsubsection{Artificial intelligence level:}

In this level commands are used for give the programming commands for the robots of controlling system when you want to execute the particular path then robots take executable correct command. where the program will accept a command such as, 'Pick up the bearing ' and decompose it into a sequence of lower level commands based on a strategic model of the task.

\subsubsection{Control mode level:}

where the motions of the system are modeled, including the dynamic interactions between the different mechanisms, trajectories planned, and grasp points selected. From this model a control strategy is formulated, and control commands issued to the next lower level.

\subsubsection{Servo system level:}

In this where actuators control the mechanism parameters using feedback of internal sensory data, and paths are modified on the basis of external sensory data. Also failure detection and correction mechanisms are implemented at this level. ${ }^{[3]}$

There also are different levels of abstraction for the robot programming languages:

\subsubsection{Guiding the systems:}

In which system the user leads the robot through the motion sensor to be performed the specific path. If we move the robots then user give the guidelines with help of executable codes.

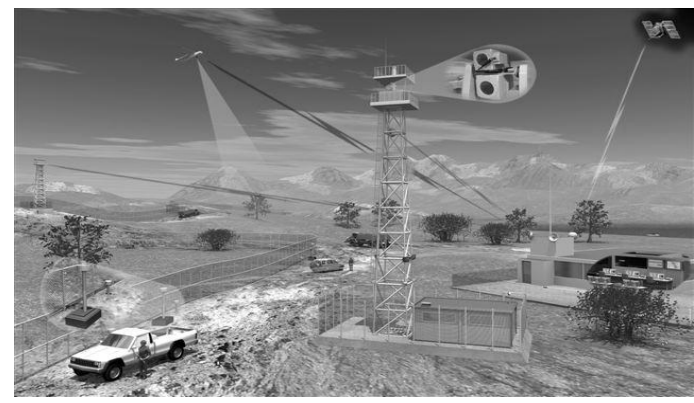

Figure 3: Advanced Monitoring System

\subsubsection{Robot-level programming:}

In which the user writes a computer program for the specify motion and tasking. In this programming we can execute the particular programming code. When we decide everything with our mind but robots decided the thing with the help of programs and he execute the programs then he jumps next levels. ${ }^{[7]}$

\section{ALGORITHM FOR SENSOR BASED ROBOTICS CONTROLLING}

The overall algorithm of the sensor-based motion planning framework is given as the following pseudo-code: ${ }^{[4]}$

$\mathrm{W} \leftarrow$ Workspace.Initialize;

$[\mathrm{C}, \Delta \mathrm{C}$ f ree, $\mathrm{C}$ unkbnd $] \leftarrow$ Cspace.Initialize $(\mathrm{W})$;

$\mathbf{R} \leftarrow$ Roadmap.Initialize $(\Delta \mathrm{C}$ f ree $) ;$

qcur $\leftarrow$ qs

it $\leftarrow 0$;

repeat

it $\leftarrow i t+1$;

path $\leftarrow$ Roadmap.Search(qcur, qg, R);

if path $!=$ NULL

qcur $\leftarrow$

Robot.Move(path);

Exit;

end;

$\mathrm{V} \leftarrow$ Fem.Solve(qs, qg, C);

$\mathrm{q}_{\mathrm{v}} \leftarrow$ Planner.NextBestView $\left(\mathrm{V}, \mathrm{C}_{\text {unkbnd }}, \mathrm{R}\right.$,

W);

path $\leftarrow$ Roadmap.Search (qcur, qv, R);

qcur $\leftarrow$ Robot.Move(path)

$\mathrm{W} \leftarrow$ Workspace.Update $(\Delta \mathrm{W}, \mathrm{W})$;

$[\mathrm{C}, \Delta \mathrm{C}$ free, Cunkbnd $] \leftarrow$ Cspace.Update $(\mathrm{C}, \mathrm{W}$ 


\author{
$\mathrm{R} \leftarrow$ Roadmap.Expand $\left(\Delta \mathrm{C}_{\text {free }}, \mathrm{R}\right) ;$ \\ until it $<=$ MAXITNUM
}

\}

In this algorithm configure the robots give the working of robots in their workplace of security system.

\section{APPLICATION OF ROBOTICS SYSTEM IN DIFFERENT TYPE OF E-GOVERNANCE TECHNOLOGY}

\subsubsection{Hospital Robotics:}

Automated robotic carts with Motivity make predictable, anywhere-to-anywhere deliveries, avoiding people and other obstacles, without expensive retro-fitting to the workplace. When vacuum tubes are too expensive, intrusive or inadequate for the job, transport systems with Motivity solve transport and conveyance problems. When manual systems put staff at risk or distract from core duties, robotic carts with Motivity provide welcome relief from dull, dirty or dangerous tasks.

\subsubsection{Remote Monitoring system in e- Governance Technology:}

Global enterprises require remote access to far-flung facilities. And in this we adopt the monitoring system to every secure areas because it is provide the monitoring system. Adept Motivity platforms collect time and spatially stamped sensor data and images for real-time viewing, historical tracking and predictive modeling of conditions at remote sites. Optional pan-tilt laser pen allows viewer to point in remote space to direct occupants' attention for remote support calls and maintenance questions. Remote monitoring system are used in defense areas and facilitate the government technology. ${ }^{[8]}$

\subsubsection{Security Robotics System in Securities Areas:}

in this user remotely monitor, manage, inspect, and assist a facility with intelligent mobile robots powered by Adept Motility by integrating them with building information and security management systems. Interrupt patrols for incident response such as alarm verification, supply delivery, or calls for resources at an event location. Implement security automation on a controlled-cost, low-impact schedule; start with one robot, build to 100 . New checkpoints, tasks, and sites can be added quickly. Typical ROI is 6-12 months in a $24 / 7$ operation, with added benefits from improved tracking, increased reliability, and lower worker risk of hazard.

\subsubsection{Outdoor Robots:}

Adept Mobile Robots outdoor platforms offer multiple solutions for commercial applications. Whether the need is a lighter, easily transported base with hot swappable batteries, or a larger base capable of running all day without recharging, Adept Mobile Robots has a Seeker robot platform for the task. ${ }^{[11]}$ Both Seeker robot platforms can be teleported, driven by a cabled joystick or allowed full autonomy, performing tasks and patrolling routes unsupervised. Add GPS, laser rangefinders, PCs, or application-specific hardware like gas sensors or powerful manipulators to get the job done in a costeffective manner without putting a human in harm's way.

\subsubsection{Intelligent Robotics Kiosks:}

Intelligent display robots with Adept Motility tote touch screen data displays, customized, networked, embedded PC and supplies where needed, avoiding people and other obstacles, ${ }^{[9]}$ without expensive retro-fitting to the workplace. The people-height touch screen makes interaction easy. When staff hands are full, mobile robots with Adept Motility can carry what's needed to the next location on their own and begin working or follow a staff-person there. ${ }^{[10]}$ Or they can guide visitors and provide tours. Implement touch screen robots on a controlled-cost, low-impact schedule; start with one robot, build to 100. Add new destinations, tasks and worksites quickly, or just stay in follow mode. Typical ROI is 12 months in a $24 \mathrm{X} 7$ operation, with added benefits from improved tracking, increased productivity and lower worker risk of hazard.

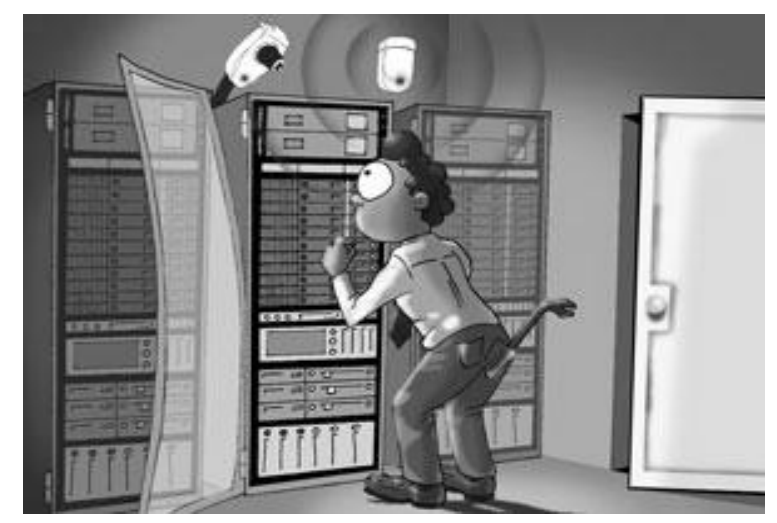

Figure 4: Robots Security System with Monitoring

\subsection{What Robots Can Do Right Now in e-}

\section{Governance Technology?}

Some Example we can show here ${ }^{[6]} \ldots \ldots$

\subsubsection{Cleaning:}

vacuum cleaner is best example of the cleaning system at our Home, even if you're not at home!

\subsubsection{Automated Hauling:}

Several robots will carry dishes and other small loads from room to room. A friend, recovering from hip surgery, to carry food from the kitchen to the living room, and the dirty dishes back into the kitchen again. Since he was on crutches, this was a real lifesaver.

\subsubsection{Security:}

Home robots could easily be tied into a computerized home security system, and the robot's mobility would allow more areas in the home to be protected. And robots make secure security system.

\subsubsection{Alarm Clock:}

With a little work I will soon be able to use Cybert as an alarm clock. Every morning he will roll into my bedroom and wake me up; once he senses that I'm out of bed he will follow me into the bathroom and deliver up-to-the minute news, weather, sports, and stock market information.

\subsubsection{Entertainment:}

Robotics is an exciting hobby for many people around the world. There are countless clubs, websites, and books that have been written for those who are interested in the topic.

\subsubsection{Education:}

Using a home robot not only teaches about robotics, it teaches spatial navigation, mapping, dud reckoning, programming, and more. 


\subsection{What Robotics System will be Able to do in the Future Technology?}

Some examples of Robotics are as follows who has used in future as ${ }^{[6]}$ :

\subsubsection{Pest Control:}

Small robots may one day scurry around our homes at night, locating and smashing cockroaches and other unwanted guests (no, I'm not talking about your mother-in-law.).

\subsubsection{Child Care:}

The technology already exists to use a robot to check on the kids while we are away from the house. Robotics will soon add a camera and an Internet interface that would allow someone to "drive" the robot around from a remote computer and receive live pictures of everything that Cye "sees". ${ }^{[7]}$

\subsubsection{Advanced Home Security and Management:}

Robots in the near future will use advanced AI (artificial intelligence) to monitor our homes, make sure everything is functioning properly and watch out for intruders.

\subsubsection{Hazard Detection:}

It would be fairly easy to attach fire, smoke, carbon monoxide, and other detectors to a home robot. Every night the robot could "make the rounds" to ensure that everything is okay. ${ }^{[12]}$

\section{FUTURE SCOPE}

in this framework we can use the advanced features for advanced security system. Because sensor based security system is the advanced technology for new generation. It has used sensor in robots and control it for many security purpose. It is provide the fast and secure security system in this framework we can handle the features of security.

\section{CONCLUSION}

In this paper we presented a new advanced architecture of sensor-based robotics algorithm of security system planning framework for e-Governance technology environments. In this algorithm technology we can implement an advanced security system with the help of robotics environments. The main idea of the proposed planning approach is to utilize a different type of security system in e-Governance. In this paper we discussed that Robots can help in different areas like Hospital, Defense, Home and others of e-Governance areas. Sensor based robotics system provide easier and fast security system and it is very useful for peoples because in this time every human can used hacking and many more technologies then these technology very helpful for e-Governance.

\section{REFERENCES}

[1] M. Kazemi and M. Mehrandezh, "Robotic navigation using harmonic function-based probabilistic roadmaps", in Proc. of the IEEE International Conference on Robotics and Automation, New Orleans, LA, Apr. 2004, pp. 4765.4770 .

[2] M. Kazemi, M. Mehrandezh, and K. Gupta, "An incremental harmonic function-based probabilistic roadmap approach to robot path planning". In submitted to the IEEE International Conference on Robotics and Automation, Barcelona, Spain, Apr. 2005.

[3] K. N. Kutulakos, V. J. Lumelsky, and C. R. Dyer, "Vision-guided exploration: A step toward general motion planning in three dimensions", in Proc. of the IEEE International Conference on Robotics and Automation, May 1993, pp. 289-296.

[4] Y. Yu and K. Gupta, "Sensor-based probabilistic roadmaps: experiments with an eye-in-hand system", Advanced Robotics, vol. 14, pp. 515-536, 2000

[5] M. Kazemi, M. Mehrandezh and Kamal Gupta. "SensorBased Robot Path Planning Using Harmonic Functionbased Probabilistic Roadmaps".

[6] S. Shapiro, ed.,"Encyclopedia of Artificial Intelligence", John Wiley \& Sons, New York, 1992.

[7] Ahmad A. Masoud, "A Hybrid, PDE-ODE Control Strategy for Intercepting an Intelligent, Well-Informed Target in a Stationary, Cluttered Environment", accepted for publication at: Applied Mathematical Sciences, HIKARI Ltd, Vol. 1, 2007, no. 48, 2345-2371.

[8] Ahmad A. Masoud, "A Discrete Harmonic Potential Field for Optimum Point-to-point Routing on a Weighted Graph", the 2006 IEEE/RSJ International Conference on Intelligent Robots and Systems", October 913 2006, Beijing-China, pp. 1779-1784.

[9] D. Keymeulen and J. Decuyper, "The fluid dynamic applied to mobile robot motion: the stream field method," in Proc. of the IEEE International Conference on Robotics and Automation, Sacramento, CA, Apr. 1994, pp. 790-796.

[10] Wiener N. "The Human Use of Human Beings: Cybernetics and Society", Houghton Mifflin Company, Boston.

[11] Wiener N. "Cybernetics, or Control and Communication in the Animal and the Machine", The MIT press, Cambridge, Massachusetts.

[12] C. I. Connolly and R. Grupen, "On the applications of harmonic functions to robotics", Journal of Robotic Systems, vol. 10, no. 7, pp. 931-946, 1993 